Onkologe 2014 · 20:1174-1176

DOI 10.1007/s00761-014-2844-6

Online publiziert: 9. November 2014

c) Springer-Verlag Berlin Heidelberg 2014

\author{
K. Höffken ${ }^{1}$ M. Bamberg ${ }^{2}$ P. M. Schlag ${ }^{3}$ \\ ${ }^{1}$ Universitätsklinikum Düsseldorf \\ 2 Universitätsklinikum Tübingen \\ ${ }^{3}$ Berlin
}

\title{
20 Jahre und kein bisschen müde
}

\section{Der Onkologe feiert Jubiläum}

Die onkologische Facharztzeitschrift Der Onkologe geht in die dritte Dekade ihres Bestehens. Wer hätte das vor 20 Jahren gedacht? Auch wenn eine Lücke in der Reihe der Fachzeitschriften des Springer-Verlags gefüllt wurde, gab es genügend skeptische Stimmen, die der Zeitschrift nur eine kurze Lebensspanne voraussagten, da sie mehr oder weniger große Schnittmengen mit anderen Fachzeitschriften, wie z. B. Der Internist, Der Chirurg und Der Radiologe, aufweisen würde. In einem Geleitwort zur ersten Zeitschriftenausgabe 1995 (- Abb. 1) verkündeten wir - die 3 Gründerherausgeber K. Höffken, M. Bamberg und P.M. Schlag - unsere Absicht, eine Fachzeitschrift aus der Taufe zu heben, die die Interdisziplinarität der Onkologie exzellent vertritt. Dieser wichtige Aspekt der interdisziplinären Themenaufstellung zieht sich seit dem Gründungsjahr wie ein roter Faden durch die Beiträge. Nach unserer Ansicht war sie bereits 1995 ein unverzichtbares Kennzeichen der modernen Onkologie - und ist es bis heute geblieben.

Was hat also noch zu dem 20-jährigen Bestehen mit guten Aussichten auf weitere Jahre der Vermittlung von onkologischem Fachwissen geführt?

$\mathrm{Da}$ ist $\mathrm{zu}$ allererst die Leserschaft $\mathrm{zu}$ nennen, die über Jahre in zunehmender Zahl ihr Interesse schenkten und dann auch die Treue hielten. Es ist der Beweis, dass die Konzeptwahl richtig war, in jedem Heft ein Schwerpunktthema zu behandeln. Komplementierende Rubriken wie „How to do?"“ oder „Repetitorium“ rundeten in der Anfangszeit die Ausgaben ab. Herausgeber und Verlag entwickelten die Zeitschrift stetig weiter. So heißen die zusätzlichen Rubriken neben dem Leitthema heute „Palliativmedizin und Supportivtherapie“ oder „Was Patienten fragen“ und bieten den Lesern eine Vielfalt an Themen in jeder Ausgabe. Seit der Gründung wurden über 2800 Beiträge in Der Onkologe veröffentlicht! Die zertifizierte Fortbildung ist seit 2001 ein wichtiger Teil der Zeitschrift. Dies macht auch die heutige Wahrnehmung unter den Abonnenten aus:

\section{》) Der Onkologe dient den onkologisch tätigen Ärztinnen und Ärzten als Fortbildungslektüre}

Einen entscheidenden Beitrag zum Erfolg der Zeitschrift leisten die engagierten Schriftleiter und die Autoren, die für die Beiträge des Schwerpunkthefts verantwortlich sind. Das onkologische Wissen wird von den Größen der deutschen onkologischen Forschungsszene zur Verfügung gestellt, die gesicherte Erkenntnisse in Übersichtsbeiträgen über ,ihre eigene" Krebsentität verfassen. Die in unterschiedlichen Abständen stattfindende Aktualisierung ermöglicht es der Leserschaft, auf dem neuesten Stand des mit einer kurzen Halbwertszeit verbundenen onkologischen Wissens zu sein.

Last but not least hat das gut kooperierende und über die Jahre eingespielte He- rausgeberteam zusammen mit den Mitarbeiterinnen der Verlagsredaktion für Kontinuität und behutsame Neuerungen gesorgt.

Wie soll die Zukunft der Zeitschrift nun aussehen? Regelmäßige Leserbefragungen geben uns wertvolle Anregungen. Dem stellen wir uns:

Wir werden Aktualität, Themenvielfalt und Interdisziplinarität auf die oberste Stufe der konzeptionellen und redaktionellen Arbeit stellen. Ferner werden wir zukünftig jüngere Kolleginnen und Kollegen um Unterstützung in der Herausgebertätigkeit bitten. Dadurch erreichen wir, innovative Ansätze aufzunehmen und gleichzeitig die Erfahrung der bisherigen

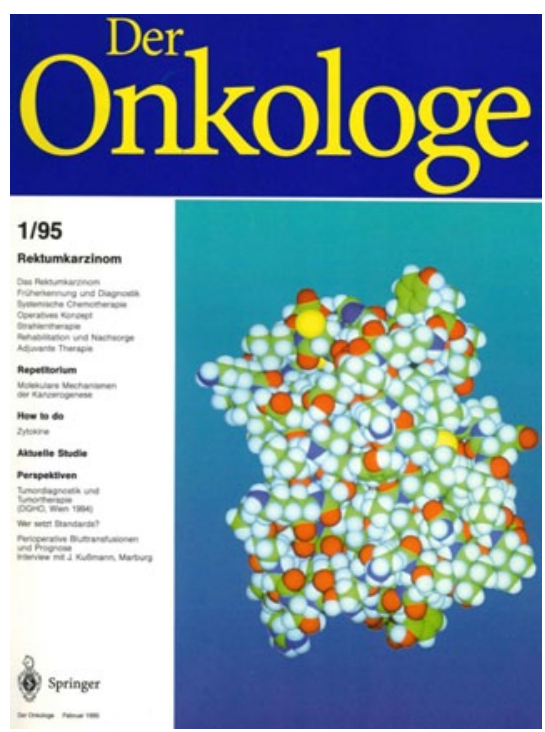

Abb. $1 \Delta$ Die erste Ausgabe von Der Onkologe startete mit dem Leitthema „Rektumkarzinom“ 
Hier steht eine Anzeige.

黛 Springer 
Herausgeber zu nutzen. Für den weiteren Erfolg der Zeitschrift sind Herausgeber und Verlag auch nach 20 Jahren kein bisschen müde geworden, das Ohr am Boden $\mathrm{zu}$ halten und auf Vorschläge und Wünsche der Leser von Der Onkologe zu achten und einzugehen.

Ihre Herausgeber-Trias<smiles>CCCC</smiles><smiles>C(=C1CC1)C1CC1Cc1ccccc1</smiles>

M. Bamberg<smiles>CCCCC(C)(C)CCC(C)(C)C</smiles>

K. Höffken

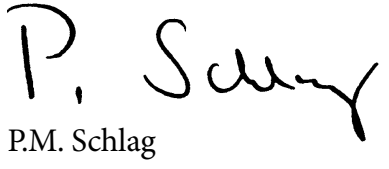

\section{Korrespondenzadresse}

\section{Prof. Dr. K. Höffken}

Universitätsklinikum Düsseldorf

Moorenstraße 5, 40225 Düsseldorf

Hoeffken.Editor@med.uni-duesseldorf.de

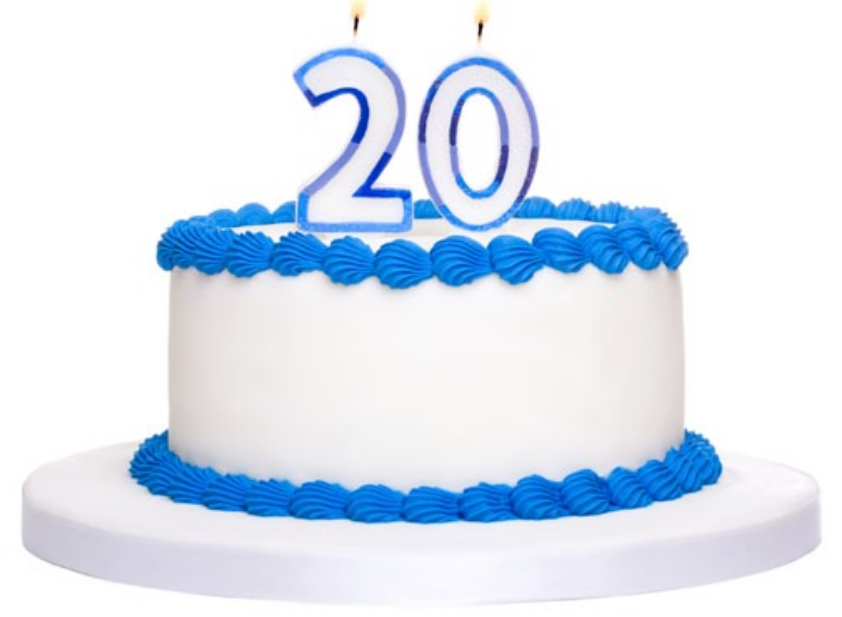

Liebe Leserinnen und Leser,

Der Onkologe feiert 20. Geburtstag!

Im Jahr 1995 wurde die Zeitschrift aus der Taufe gehoben mit dem Ziel, umfassendes onkologisches Fachwissen zu vermitteln. Wir danken unseren Herausgebern und Autoren, den Gutachtern und Ihnen, verehrte Leserinnen und Leser, für Ihr Interesse und Ihre Unterstützung. Um die Zeitschrift auch weiterhin an Ihre Bedürfnisse anzupassen, haben wir im vergangenen Jahr neue Rubriken eingeführt. Bitte schreiben Sie uns bis zum 15.02.2015 Ihre Meinung zu den Rubriken "Was Patienten fragen" und "Palliativmedizin und Supportivtherapie".

Bitte per E-Mail an:

gewinnspiel-onkologe@springer.com

Nur durch Ihr Feedback können wir Der Onkologe noch besser machen. Wir freuen uns auf Ihre Anregungen!

Unter den eingehenden Nachrichten verlosen wir 6 Präsente:

\section{1.-3. Preis}

$3 x$ ein Jahresabonnement e.Med das Kombi-Abo für alle Ärzte

Mit dem Kombi-Abo "e.Med" greifen Sie unter springermedizin.de digital auf alle Inhalte zu, die Sie als Arzt für Ihren Praxis- oder Klinikalltag brauchen. e.Med bietet Ihnen unbeschränkten Onlinezugriff auf über 500 zertifizierte Fortbildungen und darüber hinaus auf alle Springer Medizin Zeitschrifteninhalte und Archive. Zusätzlich erhalten Sie im e.Med-Abo eine gedruckte Fachzeitschrift Ihrer Wahl inklusive. Diese können Sie aus insgesamt 80 Springer Medizin Fachzeitschriften auswählen.

Wert: je $399 €$
4. Preis: Hämatologische Erkrankungen. Atlas und diagnostisches Handbuch. Löffler $\mathrm{H}$, Haferlach, T (2013)

2., überarb. Aufl. Wert: 119,99€

\section{Preis}

Knochenmetastasen. Pathophysiologie, Diagnostik und Therapie. Unter Mitarbeit von Todenhöfer T. Stenzl A, Fehm T, Hofbauer LC, Jakob, F (Hrsg., 2014) Wert: $46,99 €$

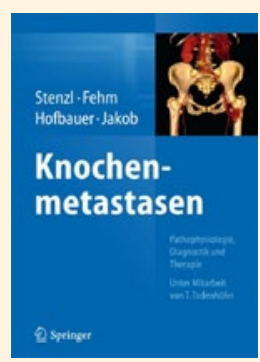

\section{Preis \\ Klinische \\ Psychoonkologie. Kusch M, Labouvie $\mathrm{H}$, Hein-Nau B (2013) \\ Wert: $39,99 €$ \\ Klinische Psycho- onkologie

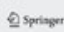 \\ Auf weitere spannende Jahre mit Ihnen freut sich Ihr Redaktionsteam Der Onkologe}

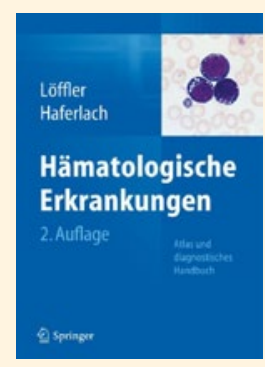

